\section{ORIGINAL RESEARCH}

\author{
C. van Pul \\ B.J.M. van Kooij \\ L.S. de Vries \\ M.J.N.L. Benders \\ A. Vilanova \\ F. Groenendaal
}

\title{
Quantitative Fiber Tracking in the Corpus Callosum and Internal Capsule Reveals Microstructural Abnormalities in Preterm Infants at Term-Equivalent Age
}

BACKGROUND AND PURPOSE: Signal-intensity abnormalities in the PLIC and thinning of the CC are often seen in preterm infants and associated with poor outcome. DTI is able to detect subtle abnormalities. We used FT to select bundles of interest (CC and PLIC) to acquire additional information on the WMI.

\begin{abstract}
MATERIALS AND METHODS: One hundred twenty preterm infants born at $<31$ weeks' gestation with 3T DTI at TEA entered this prospective study. Quantitative information (ie, volume, length, anisotropy, and $\mathrm{MD}$ ) was obtained from fiber bundles passing through the PLIC and CC. A general linear model was used to assess the effects of factor (sex) and variables (GA, BW, HC, PMA, and WMI) on FT-segmented parameters.
\end{abstract}

RESULTS: Seventy-two CC and 85 PLIC fiber bundles were assessed. For the CC, increasing WMI and decreasing FA $(P=.038)$, bundle volume $(P<.001)$, and length $(P=.001)$ were observed, whereas MD increased $(P=.001)$. For PLIC, MD increased with increasing WMI $(P=.002)$. Higher anisotropy and larger bundle length were observed in the left PLIC compared with the right $(P=.003, P=.018)$.

CONCLUSIONS: We have shown that in the CC bundle, anisotropy was decreased and diffusivity was increased in infants with high WMI scores. A relation of PLIC with WMI was also shown but was less pronounced. Brain maturation is affected more if birth was more premature.

ABBREVIATIONS: $\mathrm{BW}=$ birth weight; $\mathrm{CC}=$ corpus callosum; $\mathrm{Cl}=$ case linear anisotropy index; $F A=$ fractional anisotropy; $F T=$ fiber tracking; $\mathrm{GA}=$ gestational age; $\mathrm{HC}=$ head circumference; $\mathrm{MD}=$ mean diffusivity; PLIC = posterior limb of the internal capsule; PMA = postmenstrual age; $\mathrm{TEA}=$ term-equivalent age; $\mathrm{WMI}=$ white matter injury

$\mathbf{P}$ remature birth of $<30$ weeks' gestation is associated with a high risk of neurodevelopmental impairments, including cognitive and motor disabilities. ${ }^{1-3}$ The underlying neuropathology for cognitive disabilities is largely unknown, though it has been suggested that disturbances in the WM maturation play a role. ${ }^{4,5}$ In teenagers, the effects of preterm birth are associated with thinning of the CC, widening of the ventricles, reduced volumes of the $\mathrm{WM}$, and microstructural changes in the PLIC and the CC. ${ }^{6-9}$

MR imaging is commonly performed in preterm infants

Received May 24, 2011; accepted after revision July 29.

From the Department of Neonatology (C.v.P., B.J.M.v.K., L.S.d.V., M.J.N.L.B., F.G.), Wilhelmina Children's Hospital/University Medical Centre Utrecht, Utrecht, the Netherlands; Department of Clinical Physics (C.v.P.), Máxima Medical Center Veldhoven, Veldhoven, the Netherlands; School of Medical Physics and Engineering (C.v.P.) and Department of Biomedical Engineering (A.V.), Eindhoven University of Technology, Eindhoven, the Netherlands.

This study was funded by the Netherlands Organisation for Health Research and Development, project 94527022

Paper previously presented in part at: Annual Meeting of the International Society for Magnetic Resonance in Medicine, May 1-7, 2010; Stockholm, Sweden.

Please address correspondence to Carola van Pul, PhD, Department of Clinical Physics, Máxima Medical Center Veldhoven, De Run 4600, PO Box 7777, 5500 MB Veldhoven, the Netherlands; e-mail: c.vanpul@mmc.nl

Indicates open access to non-subscribers at www.ajnr.org

Indicates article with supplemental on-line table.

Indicates article with supplemental on-line figure.

http://dx.doi.org/10.3174/ajnr.A2859 around TEA to detect brain injury. Different scoring systems have been developed to quantify MR imaging findings. ${ }^{2,10} \mathrm{Al}-$ though these scoring systems were related to outcome, they did not provide information about the pathology on a microstructural level. WM maturation may best be followed with DTI because this technique uses both information on the diffusion properties of water molecules that are restricted in tissue and information on the direction of diffusion (anisotropy). DTI has been shown to be sensitive to microstructural WM changes, like myelination. ${ }^{11,12}$ Most information concerning DTI changes in relation to brain maturation is available from studies assessing selected ROIs in the WM. ${ }^{11}$ It has been shown that the anisotropy in preterm infants is decreased at TEA in certain brain regions (among these, the CC and the frontal WM) compared with term-born controls. ${ }^{13}$

Instead of measuring local effects in selected ROIs, FT can be applied to measure changes in bundles of WM. FT is a 3D visualization technique that reconstructs the underlying linear structure defined by the diffusion tensor. ${ }^{14}$ Quantification of $\mathrm{WM}$ tracts in infants has been performed previously ${ }^{15-23}$ and is based on calculating an average of a certain DTI parameter over the complete bundle. Axial and radial diffusivity over specific tracts have been shown to be differentially affected by bundle maturation with age, ${ }^{20,21}$ and DTI parameters quantifying the corticospinal tract are affected in preterm infants with WMI. ${ }^{22}$ Only 1 study recently investigated the genu and splenium of the CC in premature infants at TEA by using FT. ${ }^{23}$ The CC is of interest because reduced callosal volumes have 
been shown to correlate with motor function and cognitive impairment. ${ }^{24,25}$

The aim of our study was to investigate whether diffusion tensor parameters, abstracted by using FT to select complete CC and PLIC bundles, display WM abnormalities in the premature infant at TEA. The WMI score ${ }^{2}$ (as measured with conventional MR imaging) was used as a reference index.

We hypothesized that WMI would be better detected with DTI and that it would give more information on the microstructure. We investigated, furthermore, how the DTI parameters were affected by GA at birth, BW, HC, sex, and the PMA, defined here as the age at the time of scanning.

\section{Materials and Methods}

\section{Study Group}

Preterm infants with GA below 31 weeks and who reached TEA between January 2007 and July 2008 were part of a prospective cohort study. This study was approved by the medical ethics committee of our institution. Informed parental consent was obtained for all neonates. Infants were included if a 3T MR imaging scan was obtained at TEA with high-resolution DTI. Neonates were excluded if they showed signs of chromosomal or syndromal abnormalities with known effects on brain development or neuromotor development or when they had an infection of the central nervous system. The infants were sedated with $50-60 \mathrm{mg} / \mathrm{kg}$ of chloral hydrate orally 15 minutes before the MR imaging, and hearing protection was used. Heart rate, transcutaneous oxygen saturation, and respiratory rate were monitored during scanning. A neonatologist was present throughout the MR imaging examination.

\section{MR Imaging Protocol}

MR imaging was performed on a $3 \mathrm{~T}$ scanner (Achieva; Philips Healthcare, Best, the Netherlands) by using an 8-channel sensitivity encoding head coil with a sagittal T1-weighted scan with TE/TR = $15 / 886 \mathrm{~ms}$ and scan voxel size $=0.78 \times 0.98 \times 3.0 \mathrm{~mm}$ (reconstruction voxel size $=0.39 \times 0.39 \times 3.0 \mathrm{~mm})$, an axial 3D T1-weighted series with $\mathrm{TE} / \mathrm{TR}=4.6 / 9.4 \mathrm{~ms}$ and scan voxel size $=0.94 \times 0.94 \times$ $2.0 \mathrm{~mm}$ (reconstruction $=0.35 \times 0.35 \times 2.0 \mathrm{~mm}$ ), and an axial T2-weighted series with TE/TR $=120 / 6293 \mathrm{~ms}$ and scan voxel size $=$ $0.54 \times 0.61 \times 2.0 \mathrm{~mm}$ (reconstruction $=0.35 \times 0.35 \times 2.0 \mathrm{~mm})$.

DTI was based on an axial single-shot EPI sequence with a sense factor of $3(\mathrm{TE} / \mathrm{TR}=48 / 7745 \mathrm{~ms}$; duration $=4: 32$ minutes $), 2 \mathrm{~b}$-values $\left(0-800 \mathrm{~s} / \mathrm{mm}^{2}\right)$ in 32 directions. Fifty sections were acquired with a scan voxel size of $1.41 \times 1.44 \times 2.0 \mathrm{~mm}$ (reconstruction $=1.41 \times$ $1.41 \times 2.0 \mathrm{~mm}$ ). Correction for eddy currents and rigid motion was performed by using the registration software of the Philips workstation. Following registration, DICOM images were converted to a format compatible with the FT program. ${ }^{26}$

\section{WM Score}

MR images were assessed by 2 neonatologists with $>10$ years' expertise in reading MR imaging ${ }^{27,28}$ and blinded to the neurodevelopmental outcome. In case of disagreement, a third reader was consulted to achieve consensus. WM signal intensity, size of the subarachnoid space, presence of cysts, size of the ventricles, and shape of the CC were scored as 1 , normal; 2 , mildly abnormal; or 3, moderately/severely abnormal (adjusted from Woodward et $\mathrm{al}^{2}$ ). The WM score was the sum of these subscores (range, 5-15) and was applied as an indicator for WMI (normal, 5-6; mildly abnormal, 7-9; moderately abnormal, 10-12; severely abnormal, 13-15).

In addition to Woodward et $\mathrm{al},{ }^{2}$ we implemented an additional criterion to score the size of the CC. The thickness was measured on the sagittal T1-weighted MR image by 1 independent researcher at 0.5 $\mathrm{cm}$ from the natural incurvation near the level of the genu and the splenium and perpendicular to the contour and middle of the CC. The CC was scored as follows: 1 , normal if both measurements were $>2.4 \mathrm{~mm}$; 2, mildly abnormal (focal thinning of the CC) if at least 1 measurement was between 1.8 and $2.3 \mathrm{~mm}$ and both measurements were $>1.7 \mathrm{~mm}$; and 3 , moderately abnormal (general thinning) if both measurements were $\leq 1.7 \mathrm{~mm}$. The cutoff values are based on an evaluation by 3 of our researchers to make the scoring more objective. For an additional analysis, we have divided our study group into 3 groups: normal CC, focal thinning of the $\mathrm{CC}$, and generalized thinning of the CC.

\section{FT}

The quality of the DTI was assessed, and infants with FA-based color maps displaying large artifacts (eg, wrong colors on color maps by motion artifacts, On-Line Fig $1 A$ ) or sense artifacts (On-Line Fig 1B) were excluded from further analysis.

FT was conducted in DTI tool (http://bmia.bmt.tue.nl/software/ dtitool/) ${ }^{26,18}$ based on deterministic tracking, by using "case linear anisotropy" $[\mathrm{CI}=(\lambda 1-\lambda 2) /(\lambda 1+\lambda 2+\lambda 3)] \mathrm{CI}<0.12$ and maximum angle $\alpha>10^{\circ}$ as stopping criteria. $\mathrm{CI}$ is less prone to continue tracing in planar anisotropy areas than FA. ${ }^{18}$

FT was performed with seeding in every voxel in the brain. Separate fiber structures (Fig $1 A$ ) were visualized by using filtering ROIs drawn manually on axial sections of the FA color map. ${ }^{18}$ The PLIC bundle (Fig $1 D$ ) was defined by those fibers passing through 2 filtering ROIs in the PLIC area, with the first ROI positioned at the anatomic level of the foramen of Monro and the second, on the adjacent section above this landmark. The ROIs were drawn roughly surrounding the bundle, but we took care that the fibers from the anterior limb of the internal capsule were not included (Fig $1 B$ ). By using 2 roughly drawn ROIs instead of 1 ROI, only fibers in the foot-head direction were selected. The ROIs for the PLIC selection were drawn on adjacent sections because we wanted to "segment" at least all information in the PLIC in all children. This procedure was followed for left and right hemispheres separately.

To generate the CC bundle (Fig $1 E$ ), we used 2 ROIs in sagittal sections around the midplane of the $\mathrm{CC}$ as filtering ROIs (Fig 1C). For the CC, the sections on which the ROIs were positioned were $3 \mathrm{sec}-$ tions $(4.2 \mathrm{~mm})$ apart. Experimentally, it was determined that $<3$ sections apart gave too many erroneously traced fibers. We have investigated, in previous studies, whether this protocol ensures the reproducibility of the fiber bundles obtained using this method. ${ }^{29,30}$

Next, the quality of the generated fiber bundle was assessed by 2 authors separately (FT experience, $>7$ and $>3$ years): If the volume of artifactual fibers was visually estimated to be disturbing the quantification (ie, volume of more than approximately $10 \%$ of the total bundle volume), the bundle was excluded from further analysis (On-Line Fig $1 C$ ). The visual estimation was performed by rotating the volume of fibers in 3D and estimating the extent of the artifacts. If no consensus was obtained, a third reader was consulted.

Quantification of the generated fiber bundles was done by taking DTI but also volumetric information from the voxels that are included in the CC and PLIC fiber bundles, as segmented by our FT method. In the next section, we will denote the quantification of these 


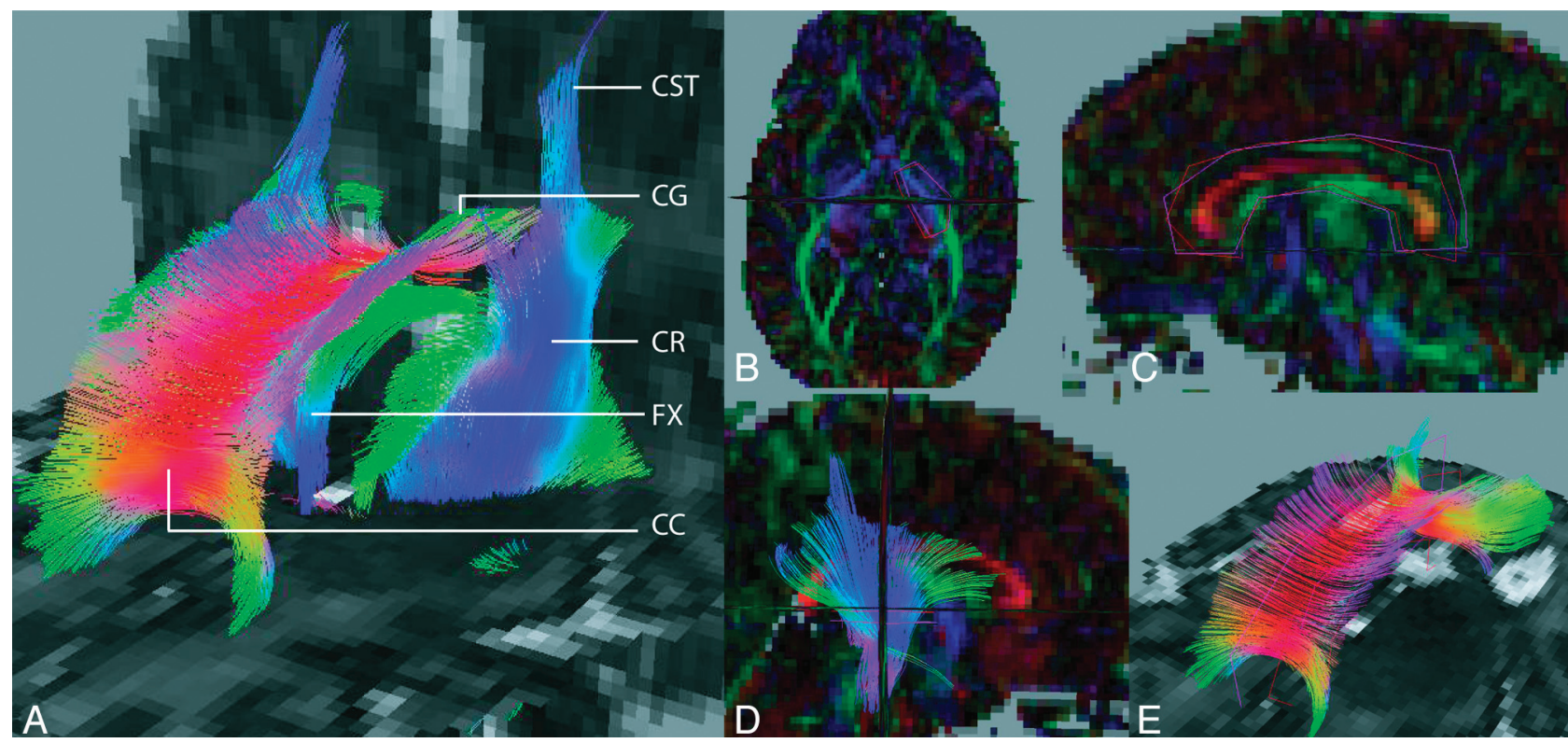

Fig 1. 3D FT superimposed on $2 D$ ADC maps. $A$, Whole-brain FT results, in a preterm infant at TEA. View from the front left. $B$, Definition of PLIC ROls. View from the top (on the axial plane). ROls superimposed on an FA-weighted color map. C, Definition of CC ROls. View from the left (on the sagittal plane). ROls superimposed on an FA-weighted color map. $D$, PLIC fiber bundle. View from the right (on the sagittal plane). ROls superimposed on an FA-weighted color map. $E$, CC fiber bundle. View from the front left (on the sagittal plane). ROIs superimposed on an ADC map. CST indicates the corticospinal tract; $C R$, the corona radiata; FX, the fornix; $C G$, the cingulum.

parameters by "FT-segmented parameters" because it includes not only DTI values but also bundle volume and length as segmented by using FT.

The bundle volume (cubic millimeters) was defined as the volume of all pixels through which $\geq 1$ fiber passed. The average bundle length (millimeter) was the average length of all fibers included in the bundle. The mean diffusivity $[\mathrm{MD}=(\lambda 1+\lambda 2+\lambda 3) / 3]\left(\mathrm{m}^{2} / \mathrm{s}\right)$, axial diffusivity $(\lambda 1)$, and radial diffusivity $[(\lambda 2+\lambda 3) / 2]$, FA, and CI of all voxels through which $\geq 1$ fiber passed were calculated.

\section{Statistical Analysis}

SPSS (Version 13.0; SPSS, Chicago, Illinois) was used for statistical analysis. To assess the relation between FT-segmented parameters and the WM score, a multivariate general linear model was used with factor (sex) and covariates (GA, PMA, HC, WM score, and BW) to determine which covariate contributed significantly to the model (model equation: DTI parameter $=\mathrm{a} 1{ }^{*} \mathrm{GA}+\mathrm{a} 2{ }^{\star} \mathrm{PMA}+\mathrm{a} 3{ }^{\star} \mathrm{HC}$ $\left.+\mathrm{a} 4{ }^{\star} \mathrm{WMI}+\mathrm{a} 5{ }^{\star} \mathrm{BW}+\mathrm{f} 1{ }^{\star} \mathrm{Sex}\right)$. The relation between FT parameter and the WM score was further evaluated by using linear regression. Differences in FT-segmented parameters among WMI groups and among WM aspects (eg, thinning of CC) were assessed by using an independent-samples $t$ test. Furthermore, differences in FT-segmented parameters between the left and right PLIC bundles were tested by using a paired-samples $t$ test. A $P$ value $<.05$ was considered statistically significant.

\section{Results}

\section{Study Group}

Of the 120 patients who entered this study, 31 were excluded because of large artifacts on imaging. Of the included 89 patients ( 48 boys, 41 girls), the mean GA was $28.5 \pm 1.7$ weeks; mean BW, $1121 \pm 338 \mathrm{~g}$; mean PMA at time of scanning, $41.7 \pm 1.1$ weeks (range, $39.6-44.7$ weeks); and mean weight at scanning of $3262 \pm 604 \mathrm{~g}$. In consensus, further exclusion of

\begin{tabular}{|c|c|c|c|}
\hline WM Aspect & $\begin{array}{c}\text { Normal } \\
\text { (score 1) }\end{array}$ & $\begin{array}{c}\text { Mildly } \\
\text { Abnormal } \\
\text { (score 2) }\end{array}$ & $\begin{array}{c}\text { Moderate/ } \\
\text { Severe } \\
\text { (score 3) }\end{array}$ \\
\hline \multicolumn{4}{|c|}{ No. of Patients with score for } \\
\hline WM signal intensity & $18(20 \%)$ & $61(69 \%)$ & $10(11 \%)$ \\
\hline Subarachnoid space & $45(51 \%)$ & $40(45 \%)$ & $4(4 \%)$ \\
\hline Presence of cysts & $87(98 \%)$ & $2(2 \%)$ & 0 \\
\hline Size of ventricles & $40(45 \%)$ & $45(51 \%)$ & $4(4 \%)$ \\
\hline Thickness of CC & $24(27 \%)$ & $52(58 \%)$ & $13(15 \%)$ \\
\hline Total WM score & $11(12 \%)$ & $66(74 \%)$ & $12^{b}(14 \%)$ \\
\hline
\end{tabular}

${ }^{\text {a }}$ For each aspect of the WM score, the number of patients in our study obtaining this score is denoted.

${ }^{\mathrm{b}}$ None had severe WM scores.

PLIC bundle tracts of 4 infants (leaving 85) and CC bundles of 17 infants (leaving 72) was deemed necessary because of artifactual FT of $>10 \%$ of the volume of the fiber bundle.

\section{WM Score}

The scoring results of the 5 aspects of WM are shown in Table 1. Eleven infants had no WM abnormalities, and most infants (74\%) had a mildly abnormal WM scores. Severe abnormalities, indicated by a WM score $>12$, were not observed in this population. In $20 \%$ of the infants, a normal WM signal intensity was observed. Most patients had a mildly abnormal signal intensity in the WM (69\%), and the abnormality was always diffuse and bilateral. Other common findings were a mildly enlarged subarachnoid space, always bilateral, and mildly enlarged ventricles. Having moderate-to-severe enlargement of both structures was rare. For the CC, the most common finding was focal thinning (58\%). One neonate showed a single small subcortical cyst frontally, and 1 child had a cyst due to a hemorrhage in the temporal lobe. During the neonatal period, 7 infants were diagnosed as having a moderate or severe intra- 

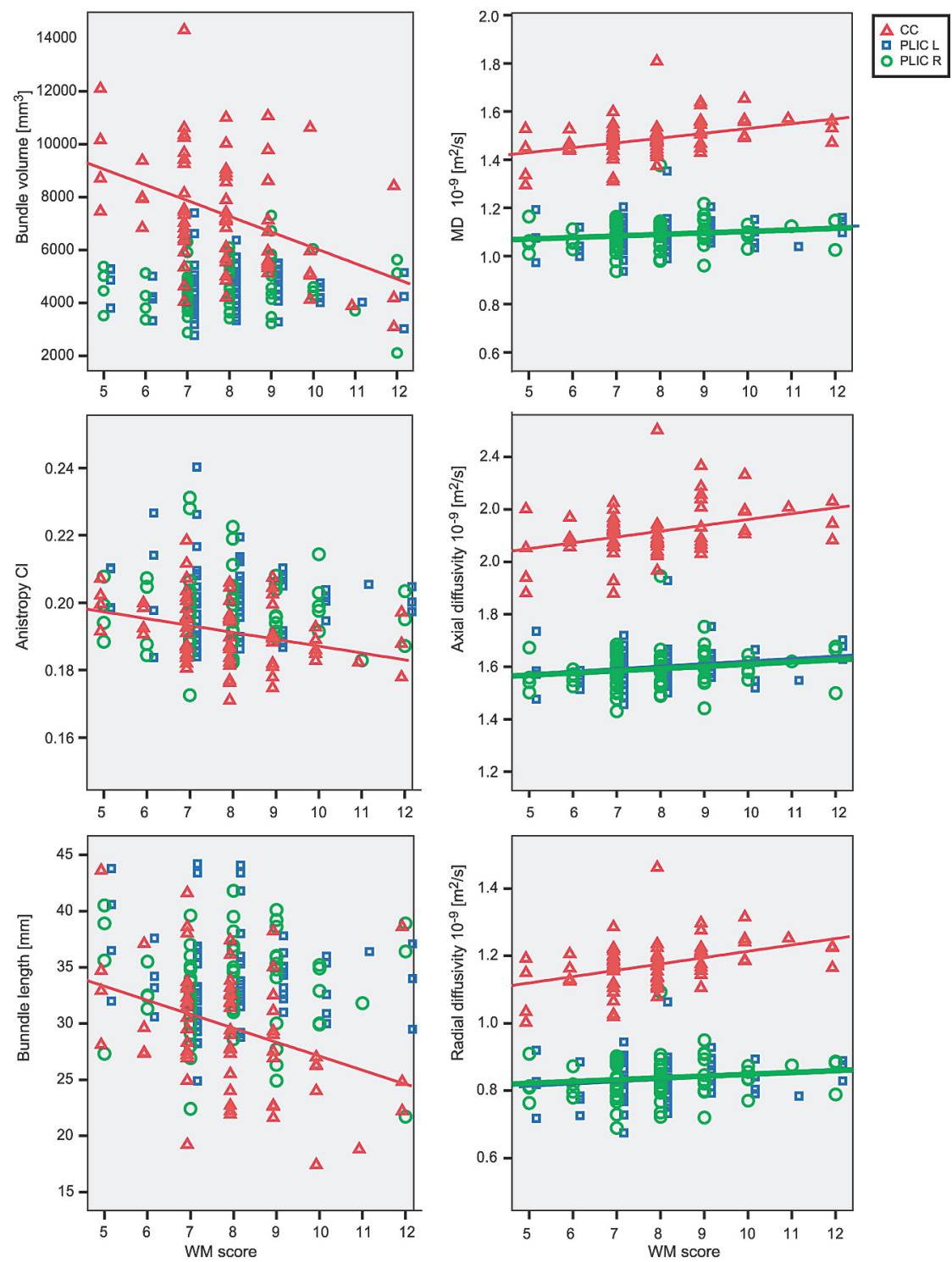

Fig 2. Bundle volume, $\mathrm{Cl}$, length, average $\mathrm{MD}$, axial diffusivity, and radial diffusivity are shown as a function of the WM score. Red triangles indicate the CC; blue squares, the left PLIC; and green circles, the right PLIC. The symbols are only for clarity and are shifted with respect to each other, to avoid cluttering of the information. The trend lines are only displayed in the graphs if the correlation was significant. For the CC, 72 bundles were evaluated and displayed in this graph, for the PLIC, 85 bundles.

ventricular hemorrhage on sequential cranial sonographic examinations, showing residual blood in the lateral ventricles on MR imaging.

\section{FT and Patient-Specific Factors}

Using a general linear model, the dependency of the FT-segmented parameters on patient-specific factors and covariates was evaluated (On-line Table). The $P$ value indicates whether the FT parameter depends significantly on the factor/covariate, taking into account the relation to the other factors/covariates. All evaluated FT-segmented parameters in the CC bundle correlated significantly with the total WM score. The GA at birth was a significant contributor to the model for the CCbundle volume and length. The PMA at time of scanning had a significant influence on the radial diffusivity and consequently also on FA and CI. The HC was an important covariate for bundle volume, length, and CI, however, not for the other FT-segmented parameters. Sex and BW (not shown) were only important for CC-bundle volume and CC-bundle length.
For the left PLIC bundle, the relation to WM score was only observed for MD and axial and radial diffusivity. Diffusivity also depended on the GA at birth and the PMA. FA and CI depended mainly on the PMA, due to the contribution of radial diffusivity.

For the right PLIC bundle, only GA, PMA, and WM scores were important for the models of the FT-segmented parameters. However, the influence was much less compared with the left PLIC bundle.

\section{FT and WM Score}

In Fig 2, FT-segmented parameters are shown as a function of the WM score. The CC-bundle volume and length were larger for infants with normal or mildly abnormal WM (low WM score) than for infants with moderately abnormal WM. Furthermore, CI decreased and MD increased with increasing WM score. For the PLIC bundles, only the diffusivity showed a significant correlation with the WM score, and the increase 

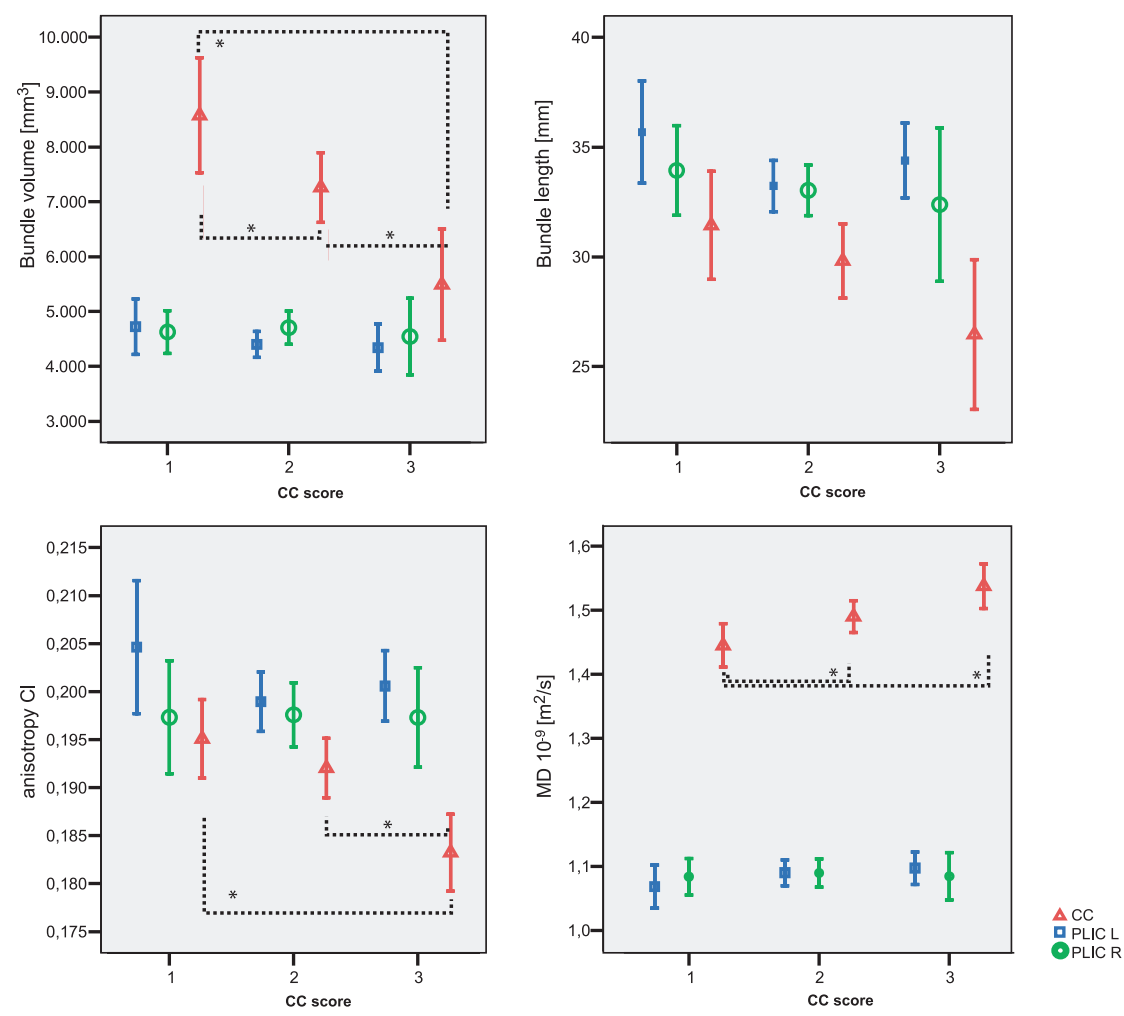

Fig 3. Average values for bundle volume, $\mathrm{Cl}$, bundle length, and average MD for the groups with CC scores 1 (normal CC), 2 (mild thinning of the CC), and 3 (severe thinning of the CC). Red triangles indicate the CC; blue squares, the PLIC L; and green open circles, the PLIC R. In total, 72 CC fiber bundles were evaluated, with $n=19$ in group $1, n=40$ in group 2 , and $n=11$ in group 3 for the CC. Displayed is the mean, and the error bar displays the standard average of the mean. Asterisk denotes a significant difference $(P<.05)$.

of the MD with increasing WM score was smaller for the PLIC bundles than for the CC bundle.

If we looked in more detail at the diffusivity, the axial diffusivity showed a significant correlation with WM for all evaluated bundles; however, the radial diffusivity only displayed a correlation for the CC and left PLIC bundle but not for the right PLIC bundle.

A larger variation in the "size characteristics" volume and length was observed for all bundles, compared with the voxelaveraged characteristics like FA, CI, and MD. In addition, the variation was larger for the CC than for the PLIC bundles.

\section{FT-Segmented Parameters and Aspect of the WM Score} Differences in FT-segmented parameters were analyzed between infants with a normal shape of the CC (score 1), focal thinning (score 2), or generalized thinning of the CC (score 3 ) (results are shown in Fig 3). Compared with the group with a normal CC, significantly reduced values for CC-bundle volume were observed in the group with focal and generalized thinning of the CC $(P=.022$ and $P=.001$, respectively $)$. In addition, CI was decreased $(P<.001)$ and $\mathrm{MD}$ increased $(P=$ $.005)$ in the CC bundle in the group with a generalized thinned CC. A trend toward decreased length was observed with increasing CC score, though it was not significant $(P=.054)$. The differences in the MD in the CC bundle between the group with a normal shape and focal thinning of the CC $(P=.032)$ were mainly due to a significant increase in radial diffusivity $(P=.027)$ and not in axial diffusivity, whereas a significant increase in both axial $(P=.028)$ and radial diffusivity $(P=$ $.027)$ was observed in the group with a generalized thinning of the CC.
In 85 infants, both the left and right PLIC bundles were eligible for analysis. A significantly higher FA and CI $(P=.002$ and $P=0.003$, respectively) and larger average length (length $=34.2 \mathrm{~mm}$ ) were observed in the left PLIC bundle compared with the right PLIC bundle (length $=33.2 \mathrm{~mm}, P=$ $.018)$.

\section{Discussion}

FT was performed to quantify CC bundles and PLIC bundles in preterm infants at TEA, to detect WMI. The CC bundle is of interest because thinning of the $\mathrm{CC}$ is commonly seen in preterm infants and is associated with a worse outcome. ${ }^{6,9}$ Not only were the CC-bundle volume and length smaller for infants with WMI but also lower anisotropy (FA and CI) was observed in combination with an increased MD, suggesting less hindered diffusion. The decrease in anisotropy was mainly driven by an increased radial diffusivity in the group with focal CC thinning, whereas both axial and radial diffusivities were increased for the group with diffuse thinning of the entire CC. Our findings were in agreement with ROI-based DTI studies showing decreased FA and increased $\mathrm{ADC}^{31}$ and radial diffusivity $^{32}$ in the CC in infants with WMI. It was suggested that changes in radial diffusion reflect myelin and premyelin effects, whereas changes in axial diffusivity are likely related to changes in axon integrity. ${ }^{22,33}$ Therefore, we hypothesized that changes in the group with focal thinning of the CC were mainly due to a slowdown in myelin or premyelin maturation, whereas the group with generalized thinning displayed larger injury, potentially including axonal injury.

This correlation of FT-segmented parameters in the CC bundle with WMI was not observed by de Bruïne et $\mathrm{al}^{23}$; how- 
ever, they evaluated the splenium and genu of the CC separately and not as an average of the CC bundles as we did.

We realize that both for CC and PLIC, the observed effect sizes in the general linear model are small, and also the slopes (Table 2) in some cases are close to zero. From the scatterplots, it is clear that this method is not yet suitable for predicting a single patient's WMI score from that same patient's anisotropy value. However, in our opinion, the information obtained from our study can be used to gain understanding in the microstructural changes resulting from premature birth.

For the left PLIC bundle, both axial and radial diffusivity increased significantly with WMI. This is in contrast to findings by Adams et al, ${ }^{22}$ showing lower FA in the corticospinal tract due to changes in radial and not in axial diffusivity. The difference might be explained by the fact that part of the corticospinal tract is included in the much larger PLIC bundle in our study.

If we compared the left and right PLIC bundles, the longer bundle length and higher anisotropy in the left PLIC bundle could reflect a better maturation compared with the right PLIC. Higher FA values in the left PLIC bundle have been observed in older children ${ }^{21}$; in neonates, larger WM volumes in the left hemisphere have been found. ${ }^{34}$

Radial diffusivity decreased and anisotropy (FA, CI) increased with increasing PMA for both structures, in agreement with findings in the literature. ${ }^{11,22,35,36}$ Furthermore, CC-bundle volume and length and right PLIC volume and length were lower and MD in the left PLIC bundle was higher for infants born at an earlier GA. Because there was no relation between GA at birth and PMA at scanning time, this finding may suggest an effect of GA on brain maturation. Maturational changes are better followed with DTI, and FT-segmented DTI parameters have been shown in young infants to give information on normal functional development and might be useful in detecting pathologic cognitive development in early infancy. ${ }^{20,21}$ Dubois et al ${ }^{20,21}$ reported that DTI metrics can be used to distinguish "premyelination" stages (ie, the isotropic proliferation of cells, prolongations, and development of intracellular compartments and membranes) and "true" myelination. Similar findings to ours were observed in adolescents born prematurely, ${ }^{8}$ with WM volume more decreased with lower GA, though a relation to GA was not observed in recent studies in preterm infants. ${ }^{22,23}$

The main limitation of our study is the large number of artifacts in the DTI acquisition. DTI is more prone to artifacts than conventional MR images. In this study, $26 \%$ of the patients had to be excluded due to motion and/or sense artifacts. The DTI sequence was at the end of the MR imaging protocol, and though the infants were sedated and hearing protection was applied, infants waking up during the DTI scan could not be prevented. Severe motion artifacts in the DTI images resulted in errors in FT, in particular when using a deterministic technique as applied in this study. To minimize the influence of errors in the quantification of the tracts, we excluded all bundles for which we agreed in consensus that the artifactual fibers contributed $>10 \%$ to the volume of the fibers.

We realize that there is a user bias in the visual interpretation of whether the erroneous traced fibers are $>10 \%$ of the total bundle volume. However, 2 users observed that by rotating the fiber structure in 3D, the visual estimation of the size of the artifacts compared with the size of the fiber bundle was possible. In case of disagreement, a third observer was consulted.

The distribution of WMI in the artifacts group was similar to that in the finally included group; therefore, no bias was introduced by excluding the patients with artifacts in the DTI data. The artifacts are not due to the WMI.

Our study shows the strength but also the weaknesses of using the complete bundle for evaluation, instead of ROIbased DTI ${ }^{31,32,36}$ or a part of the bundle. ${ }^{22,23}$ The strength is that a complete structure is evaluated and not only a local effect, making the method less sensitive to ROI-positioning errors. On the other hand, small effects may be missed because they are lost in the variances in the large bundle.

Another limitation of our study is that manually drawn ROIs were used for bundle selection, though our FT was seeded in all voxels in the brain. Our method was different from a "single ROI" method because by using 2 roughly drawn ROIs instead of $1 \mathrm{ROI}$, only fibers in the foot-head direction were selected and the method was less influenced by the exact drawing of the ROIs. The PLIC ROIs were chosen in adjacent sections because we wanted to "segment" at least all information in the PLIC in all children because WMI frequently occurs in the WM areas adjacent to the ventricles. Therefore, we did not draw the second ROI on a level $>4$ sections higher; otherwise we would not be able to trace any fibers. The reproducibility of our method was analyzed, and with the protocolized ROI definition, generated bundles were visually similar and also quantitatively comparable. However, problems with FT still occurred in areas of crossing fibers because deterministic tracking does not trace well in these areas ${ }^{14}$; this problem is inherent to the use of only the diffusion tensor and not high angular information.

Furthermore, the DTI model could not represent adequately $>1$ fiber population within a voxel. This problem could be improved by using other models in which this is possible, like Q-Ball or spheric deconvolution models; however, these models require high-angular acquisitions with more directions than what we can provide now for neonates due to the time constrains in scanning.

Therefore tracking was stopped in areas of crossing fibers, where $\mathrm{CI}$ is below the stopping value. We realize that the choice of stopping criteria influences bundle volume and length; however, within our study, differences in bundle FTsegmented parameters between groups can be compared because the same stopping criteria were used.

Compared with conventional MR imaging, quantitative FT gives microstructural information, which might give insight into understanding the pathology with subsequent poor outcome.

\section{Conclusions}

We have shown that in the FT-selected CC bundle, the anisotropy (FA, CI) was decreased and diffusivity was increased in infants with high WMI scores. Particularly, increased radial diffusivity was observed in infants with focal CC thinning, and both axial and radial diffusivities were increased in infants with generalized thinning of the CC, suggesting a difference in underlying WM pathology. A relation of the PLIC with WMI was also shown, but it was less pronounced, suggesting that the 
CC is more prone to WMI in the maturing brain than the PLIC. The volume of the CC and right PLIC bundles as determined with FT was affected by the degree of preterm birth (GA relation), suggesting an influence on brain maturation. FT additionally gives information on changes in bundle length and volume, which also decreased in infants with WMI.

Disclosures: Linda de Vries—UNRELATED: Royalties: Book: Govaert P, de Vries LS: Atlas of Neonatal Ultrasonography (John Wiley \& Sons, 2010). Floris Groenendaal—UNRELATED: Expert Testimony. expert witness in legal cases of malpractice, Travel/Accommodations/ Meeting Expenses Unrelated to Activities Listed: invited speaker for scientific meeting in Sweden, April 2010.

\section{References}

1. Bhutta AT, Cleves MA, Casey PH, et al. Cognitive and behavioral outcomes of school-aged children who were born preterm: a meta-analysis. JAMA 2002; 288:728-37

2. Woodward LJ, Anderson PJ, Austin NC, et al. Neonatal MRI to predict neurodevelopmental outcomes in preterm infants. N Engl J Med 2006;355:685-94

3. Latal B. Prediction of neurodevelopmental outcome after preterm birth. $\mathrm{Pe}$ diatr Neurol 2009;40:413-19

4. Counsell SJ, Boardman JP. Differential brain growth in the infant born preterm: current knowledge and future developments from brain imaging. Semin Fetal Neonatal Med 2005;10:403-10

5. Dudink J, Kerr JL, Paterson K, et al. Connecting the developing preterm brain. Early Hum Dev 2008;84:777-82

6. Stewart AL, Rifkin L, Amess PN, et al. Brain structure and neurocognitive and behavioural function in adolescents who were born very preterm. Lancet 1999;353:1653-57

7. Nagy Z, Westerberg H, Skare S, et al. Preterm children have disturbances of white matter at 11 years of age as shown by diffusion tensor imaging. Pediatr Res 2003;54:672-79

8. Nagy Z, Ashburner J, Andersson J, et al. Structural correlates of preterm birth in the adolescent brain. Pediatrics 2009;124:e964-72

9. Skranes J, Vangberg TR, Kulseng S, et al. Clinical findings and white matter abnormalities seen on diffusion tensor imaging in adolescents with very low birth weight. Brain 2007;130:654-66

10. Childs AM, Ramenghi LA, Cornette L, et al. Cerebral maturation in premature infants: quantitative assessment using MR imaging. AJNR Am J Neuroradiol 2001;22:1577-82

11. Neil J, Miller J, Mukherjee P, et al. Diffusion tensor imaging of normal and injured developing human brain: a technical review. NMR Biomed 2002;15: 543-52

12. Hüppi PS, Dubois J. Diffusion tensor imaging of brain development. Semin Fetal Neonatal Med 2006;11:489-97

13. Hüppi PS, Maier SE, Peled S, et al. Microstructural development of human newborn cerebral white matter assessed in vivo by diffusion tensor magnetic resonance imaging. Pediatr Res 1998;44:584-90

14. Mori S, van Zijl PC. Fiber tracking: principles and strategies. NMR Biomed 2002;15:468-80

15. Partridge SC, Mukherjee P, Berman JI, et al. Tractography-based quantitation of diffusion tensor imaging parameters in white matter tracts of preterm newborns. J Magn Reson Imaging 2005;22:467-74

16. Berman JI, Mukherjee P, Partridge SC, et al. Quantitative diffusion tensor MRI fiber tractography of sensorimotor white matter development in premature infants. Neuroimage 2005;27:862-71

17. Berman JI, Glass HC, Miller SP, et al. Quantitative fiber tracking analysis of the optic radiation correlated with visual performance in premature newborns. AJNR Am J Neuroradiol 2009;30:120-24

18. van Pul C, Buijs J, Vilanova A, et al. Infants with perinatal hypoxic ischemia: feasibility of fiber tracking at birth and 3 months. Radiology 2006;240:203-14

19. Dubois J, Hertz-Pannier L, Dehaene-Lambertz G, et al. Assessment of the early organization and maturation of infants' cerebral white matter fiber bundles: a feasibility study using quantitative diffusion tensor imaging and tractography. Neuroimage 2006;30:1121-32

20. Dubois J, Dehaene-Lambertz G, Perrin M, et al. Asynchrony of the early maturation of white matter bundles in healthy infants: quantitative landmarks revealed noninvasively by diffusion tensor imaging. Hum Brain Mapp 2008: 29:14-27

21. Dubois J, Hertz-Pannier L, Cachia A, et al. Structural asymmetries in the infant language and sensori-motor networks. Cereb Cortex 2009;19:414-23. Epub 2008 Jun 17

22. Adams E, Chau V, Poskitt KJ, et al. Tractography-based quantitation of corticospinal tract development in premature newborns. J Pediatr 2010;156: 882-88

23. de Bruïne FT, van Wezel-Meijler G, Leijser LM, et al. Tractography of developing white matter of the internal capsule and corpus callosum in very preterm infants. Eur Radiol 2011;21:538-47

24. Rademaker KJ, Lam JN, Van Haastert IC, et al. Larger corpus callosum size with better motor performance in prematurely born children. Semin Perinatol 2004;28:279-87

25. Caldú X, Narberhaus A, Junqué C, et al. Corpus callosum size and neuropsychologic impairment in adolescents who were born preterm. J Child Neurol 2006;21:406-10

26. Vilanova A, Berenschot G, Pul van C. DTI visualization with streamsurfaces and evenly-spaced volume seeding. In: Proceedings of the Joint EurographicsIEEE TCVG Symposium on Visualization. Konstanz, Germany, May 16-21, 2004

27. de Vries LS, Groenendaal F. Neuroimaging in the preterm infant. Ment Retard Dev Disabil Res Rev 2002;8:273-80

28. de Vries LS, Groenendaal F. Patterns of neonatal hypoxic-ischaemic brain injury. Neuroradiology 2010;52:555-66

29. Palen van der RL, Buijs J, Vilanova A, et al. Quantitative fiber tracking after perinatal hypoxic-ischemia and neurodevelopmental outcome at 2 years. In: Proceedings of the 16th Annual Meeting of the International Society for Magnetic Resonance in Medicine, Toronto, Ontario, Canada. May 3-9, 2008

30. Palen van der RL, van Mierlo E, Buijs J, et al. Quantitative tractography: the influence of chosen stopping criteria on the quantification parameters. In: Proceedings of the 17th Annual Meeting of the International Society for Magnetic Resonance in Medicine, Antwerp, Belgium. November 23-27, 2008

31. Skiöld B, Horsch S, Hallberg B, et al. White matter changes in extremely preterm infants, a population-based diffusion tensor imaging study. Acta Paediatr 2010;99:842-49

32. Counsell SJ, Shen Y, Boardman JP, et al. Axial and radial diffusivity in preterm infants who have diffuse white matter changes on magnetic resonance imaging at term-equivalent age. Pediatrics 2006;117:376-86

33. Song SK, Sun SW, Ramsbottom MJ, et al. Dysmyelination revealed through MRI as increased radial (but unchanged axial) diffusion of water. Neuroimage 2002;17:1429-36

34. Gilmore JH, Lin W, Corouge I, et al. Early postnatal development of CC and corticospinal white matter assessed with quantitative tractography. AJNR Am J Neuroradiol 2007;28:1789-95

35. Gilmore JH, Lin W, Prastawa MW, et al. Regional gray matter growth, sexual dimorphism, and cerebral asymmetry in the neonatal brain. $J$ Neurosci 2007;27:1255-60

36. Mathur AM, Neil JJ, Inder TE. Understanding brain injury and neurodevelopmental disabilities in the preterm infant: the evolving role of advanced magnetic resonance imaging. Semin Perinatol 2010;34:57-66 